\title{
THE EFFECT OF ACETYLCHOLINE UPON RESLIRATORY GAS EXCHANGE IN MITRAL STENOSIS
}

\author{
By J. M. BISHOP, P. HARRIS, M. BATEMAN * And L. A. G. DAVIDSON \\ (From the Department of Medicine, University of Birmingham, at the Queen Elizalicth \\ Hospital, Birmingham, England)
}

(Submitted for publication June 27, 1960; accepted August 11, 1960)

Most patients with mitral stenosis show hemodynamic evidence of an increased resistance to blood flow through the lungs. Structural abnormalities in the small pulmonary arteries are seen in such patients, with increased thickness of the vessel wall and narrowing of the lumen. The increased resistance is also in part due to active vasoconstriction in many patients, and this can be released by acetylcholine (1-3). Söderholm and Werkö (4) also observed that the oxygen saturation of arterial blood decreased during the infusion of acetylcholine in patients with mitral stenosis. They suggested that this was probably due to the increased perfusion of poorly ventilated alveoli which had previously been poorly perfused.

The present studies were undertaken in order to investigate the above possibility further by the measurement of blood gas tensions. Observations have been made while the patients breathed a gas mixture containing from 45 to 50 per cent oxygen. this level of oxygen being selected for two reasons. Firstly, the magnitude of the difference between alveolar and arterial oxygen tension (A-a difference) is greater, and hence it can be measured with a relatively greater precision. Secondly, with this concentration of inspired oxygen the "membrane component" is negligible. A subsidiary purpose of the study was the comparison of the pulmonary hemodynamics in these patients when they breathed air and when they breathed 45 to 50 per cent oxygen.

\section{METHODS}

All the patients had rheumatic heart disease with mitral stenosis, and their personal details are given in Table I. Patients were studied in the supine position, unsedated and having taken no food since a light breakfast 5 hours carlier.

A double-lumen cardiac catheter was passed into the lung so that the terminal orifice was wedged in the

* In receipt of a research grant from the Medical Research Council. periphery while the proximal orifice lay in the pulmonary artery. The tip of another single-lumen catheter was introduced into the middle of the right atrium. An indwelling needle was placed in the brachial artery. Intravascular pressures were recorded by capacitance manometers and a direct writing recorder. Mean pressures were obtained by planimetric integration over at least 2 respiratory cycles.

The percentage oxygen saturation of blood samples was estimated by a spectrophotometric method (5), and the blood oxygen capacity was measured photometrically. Gas analysis was by the Scholander micro-method. Inspired gas mixtures other than air were supplied from cylinders, the patient breathing from an anesthesia bag which served as a reservoir.

The oxygen tension of arterial blood was measured polarographically with a Clark electrode incorporated in an assembly with a magnetic stirrer (6). Each reported value of oxygen tension represents the mean of estimates on 3 blood samples. Carbon dioxide tension was measured by a modification of the interpolation method of Åstrup (7) using a Metrohm EA 125 glass electrode. All measurements were made in a water bath at $38^{\circ} \mathrm{C}$. Two syringes of blood were centrifuged at $38^{\circ} \mathrm{C}$ and a reading of plasma $\mathrm{pH}$ was made on each. The plasma was then equilibrated by bubbling with 3 gas mixtures of known carbon dioxide content and the resulting $\mathrm{pH}$ values recorded. The initial plasma $\mathrm{P}_{\mathrm{CO}_{2}}$ was determined graphically from the relationship between $\mathrm{pH}$ and $\mathrm{P}_{\mathrm{co}_{2}}$. Using this method in 16 tonometer experiments, the mean difference between measured and tonometer values was $0.6 \mathrm{~mm} \mathrm{Hg}$ ( $\mathrm{SD} \mathrm{1.2)}$.

The same protocol was followed 3 times in each patient with intervals of 15 minutes between each. In one, the patient breathed air and in another a previously analyzed mixture containing from 45 to 50 per cent oxygen in nitrogen (subsequently referred to as 47 per cent oxygen). On the other occasion the patient breathed the same oxygen-enriched gas mixture but at Minute 11 an infusion of acetylcholine into the right atrium was commenced at the rate of $2.0 \mathrm{mg}$ per minute and continued to the end of the protocol. The 6 permutations of these 3 protocols were placed in random order and allocated to the patients in the order in which they were studied. The 6 experimental plans were then repeated in the same random order, a total of 12 patients being investigated.

Each protocol lasted 25 minutes and the mouthpiece and noseclip were in place throughout. Expired gas was collected and 3 samples of blood from the brachial and 
TABLE I

Personal and clinical details of patients studied *

\begin{tabular}{|c|c|c|c|c|c|c|c|c|c|}
\hline Patient & Age & Sex & Weight & Height & SA & Rhythm & \multicolumn{2}{|c|}{$\begin{array}{l}\text { Valvular } \\
\text { lesions }\end{array}$} & Other clinical features \\
\hline F.A. & 58 & $\mathrm{~F}$ & 54 & 155 & 1.51 & $\mathrm{AF}$ & MS & MI & \\
\hline J.Ly. & 49 & M & 66 & 168 & 1.71 & $\mathrm{SR}$ & MS & & \\
\hline D.D. & 25 & $\mathrm{~F}$ & 63 & 165 & 1.68 & $\mathrm{SR}$ & MS & $\mathrm{AI}$ & \\
\hline E.R. & 37 & $\mathrm{~F}$ & 60 & 163 & 1.63 & $\mathrm{AF}$ & MS & MI & Recurrent bronchitis \\
\hline C.W. & 25 & $\mathrm{~F}$ & 57 & 160 & 1.58 & $\mathrm{SR}$ & MS & & $\begin{array}{l}\text { Pulmonary infarct } 2 \\
\text { months earlier }\end{array}$ \\
\hline D.R. & 40 & $\mathrm{~F}$ & 57 & 163 & 1.58 & $\mathrm{SR}$ & MS & & $\begin{array}{l}\text { Mitral valvotomy } 4 \\
\text { years earlier; re- } \\
\text { current bronchitis }\end{array}$ \\
\hline L.M. & 48 & $\mathrm{~F}$ & 48 & 155 & 1.24 & $\mathrm{AF}$ & MS & MI & $\begin{array}{l}\text { Mitral valvotomy } 5 \\
\text { years earlier; re- } \\
\text { current bronchitis }\end{array}$ \\
\hline M.McP. & 45 & $\mathrm{~F}$ & 59 & 155 & 1.57 & SR & MS & & $\begin{array}{l}\text { Splenectomy for } \\
\text { acholuric jaundice } \\
2 \text { years earlier }\end{array}$ \\
\hline P.K. & 30 & $\mathrm{~F}$ & 50 & 163 & 1.51 & $\mathrm{AF}$ & MS & M I & \\
\hline E.M. & 49 & $\mathbf{M}$ & 62 & 173 & 1.72 & $\mathrm{SR}$ & MS & & Recurrent bronchitis \\
\hline J.La. & 49 & M & 79 & 170 & 1.90 & SR & MS & & $\begin{array}{l}\text { Mitral valvotomy } 4 \\
\text { years earlier; prob- } \\
\text { able pulmonary } \\
\text { infarct } 1 \text { year earlier }\end{array}$ \\
\hline N.T. & 46 & $\mathrm{~F}$ & 48 & 163 & 1.50 & $\mathrm{AF}$ & MS & MI & Recurrent bronchitis \\
\hline
\end{tabular}

* Abbreviations used $: \mathrm{SA}=$ surface area $; \mathrm{AF}=$ auricular fibrillation, $\mathrm{SR}=$ sinus rhythm; $\mathrm{MS}=$ mitral stenosis, $\mathrm{MI}=$ mitral insufficiency, $\mathrm{AI}=$ aortic insufficiency.

pulmonary arteries were taken for estimation of the cardiac output between Minutes 15 and 18. Exercise on a constant rate bicycle ergometer commenced at Minute 19 and the cardiac output was again measured between Minutes 23 and 25 . Intravascular pressures were recorded immediately before and after each estimation of cardiac output. In each of the 2 protocols in which the high oxygen mixture was breathed, but not in the air-breathing protocol, arterial blood was also taken during the expired gas collection for estimation of gas tensions. Three samples for oxygen tension and two for carbon dioxide tension were taken on each occasion, and sampling extended over most of the period of gas collection.

In calculating cardiac output when oxygen-enriched mixtures were breathed, the arterial oxygen content was taken as equal to the blood oxygen capacity, plus the oxygen in physical solution, which was obtained from the measured oxygen tension and the solubility coefficient of oxygen in blood (8). In a small number of instances this procedure may have led to a slight overestimate of the arterial oxygen content when the arterial oxygen tension was below $150 \mathrm{~mm} \mathrm{Hg}$, since knowledge of the form of the hemoglobin oxygen dissociation curve at oxygen tensions greater than $100 \mathrm{~mm} \mathrm{Hg}$ is inadequate to permit the necessary correction for a possible slight unsaturation. Mixed venous blood oxygen content was cal- culated from the measured oxygen saturation and the blood oxygen capacity. The alveolar oxygen tension was calculated from the alveolar equation. Physiological dead space was estimated in the usual manner, allowance being made for an apparatus dead space of $80 \mathrm{ml}$.

The significance of differences between values when breathing air and when breathing 45 to 50 per cent oxygen, and between values when breathing 45 to 50 per cent oxygen with and without the infusion of acetylcholine, have been determined by the use of the $t$ test (Tables II and III).

\section{RESULTS}

The results are given in Tables II and III and will be described in two sections. The first concerns the observations on blood and alveolar gas tensions, and the second comprises the hemodynamic and related measurements.

\section{Arterial blood and alveolar gas tensions and de- rived measurements}

Control values at rest, breathing 47 per cent oxygen. The mean alveolar oxygen tension was $291 \mathrm{~mm} \mathrm{Hg}$ (range 277 to 316), the mean arterial 


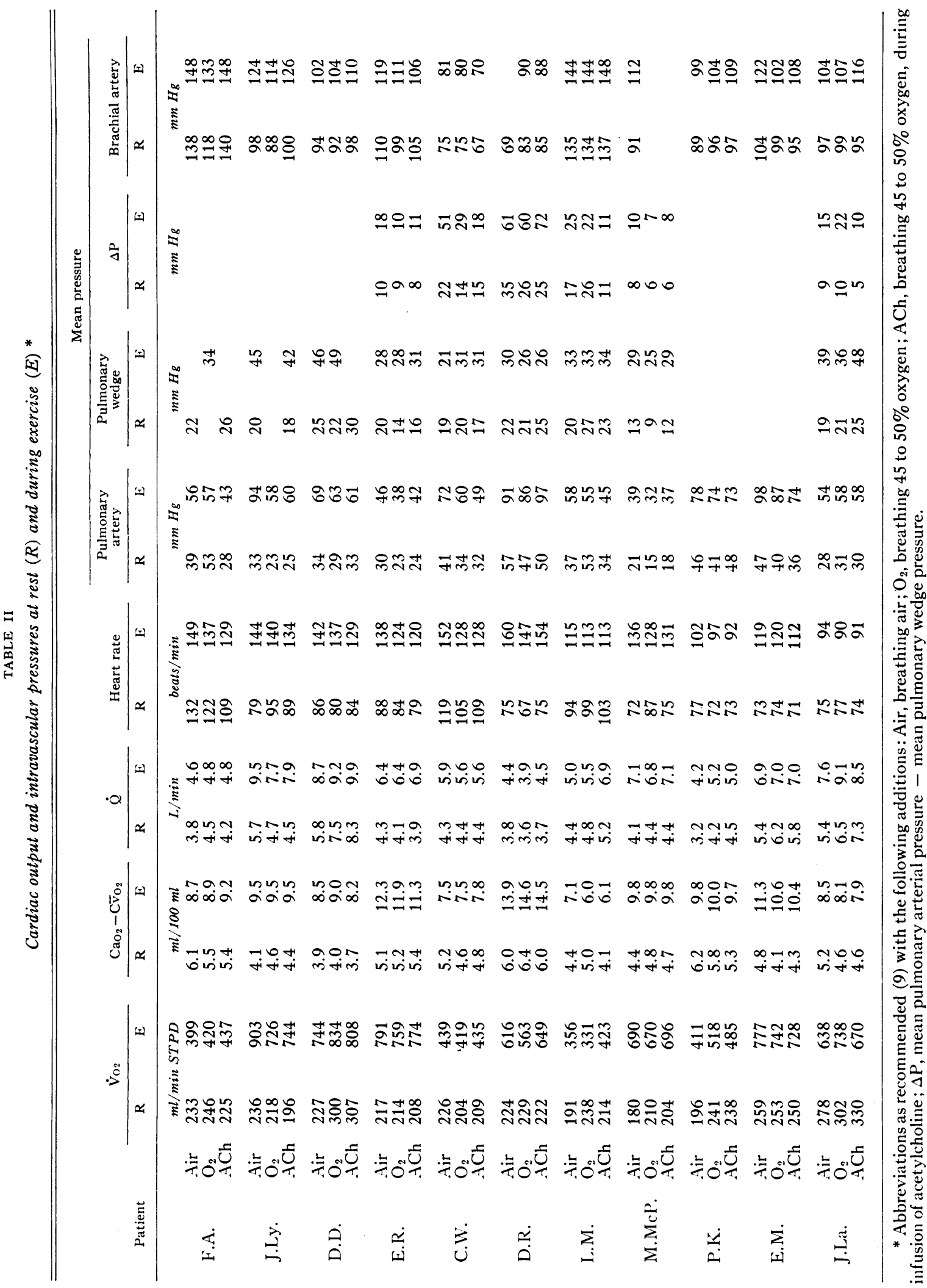




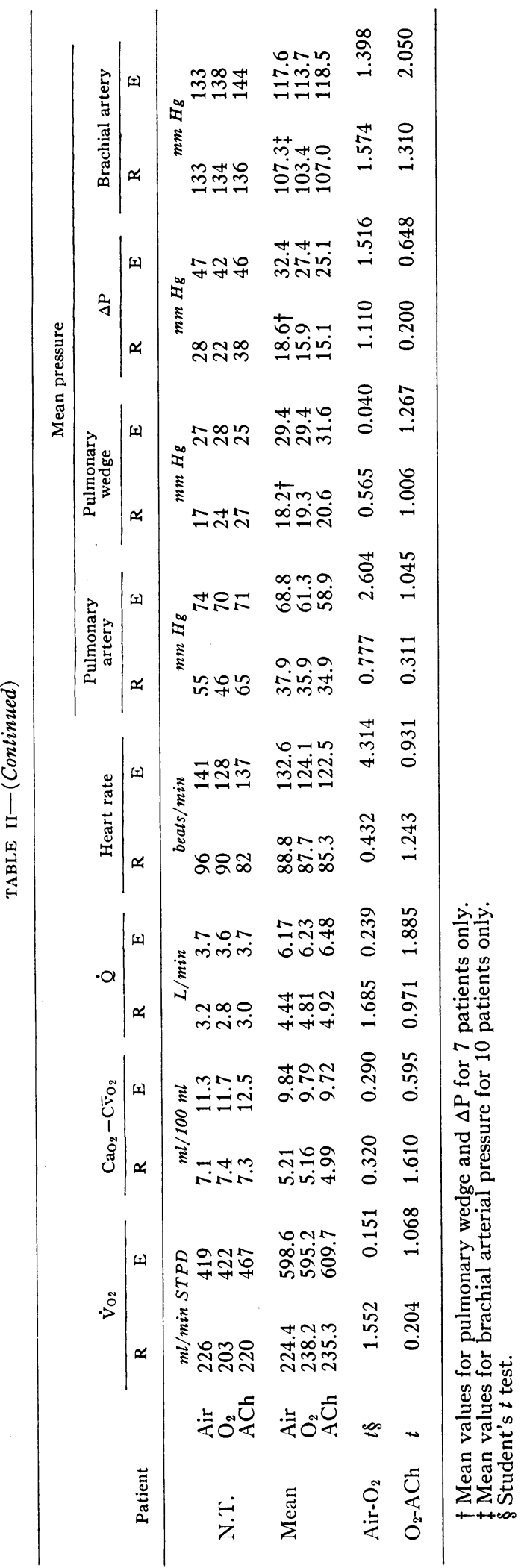

blood oxygen tension was $200 \mathrm{~mm} \mathrm{Hg}$ (range 142 to 275), and the mean A-a difference was $92 \mathrm{~mm}$ $\mathrm{Hg}$ (range 39 to 138 ).

The mean arterial carbon dioxide tension was $39.7 \mathrm{~mm} \mathrm{Hg}$ (range 32.1 to 45.2 ). The mean value for physiological dead space was $123 \mathrm{ml}$ with a range from 78 to 208 . The physiological dead space/tidal volume ratio $\left(\mathrm{V}_{\mathbf{D}} / \mathrm{V}_{\mathbf{T}}\right)$ had a mean value of 30.0 per cent (range from 21.2 to 40.9).

Effect of exercise, breathing 47 per cent oxygen. The mean alveolar oxygen tension was not appreciably different from the value at rest, and the greatest individual change was $10 \mathrm{~mm} \mathrm{Hg}$. The mean arterial oxygen tension was slightly but insignificantly less than at rest. The resultant A-a difference had a mean value which was a little greater than at rest.

The mean arterial carbon dioxide tension rose to $41.8 \mathrm{~mm} \mathrm{Hg}$ during exercise. The physiological dead space increased considerably to a mean of $190 \mathrm{ml}$, but there was only a small increase in the ratio $\mathrm{V}_{\mathrm{D}} / \mathrm{V}_{\mathrm{T}}$, which had a mean value of 31.3 per cent.

The changes in A-a difference and in the ratio $\mathrm{V}_{\mathbf{D}} / \mathrm{V}_{\mathbf{T}}$ during exercise varied in the individual patients. In general, both changed in the same direction in a particular patient and both tended to increase more consistently in patients with the higher pulmonary arterial pressures. The ratio $\mathrm{V}_{\mathbf{D}} / \mathrm{V}_{\mathbf{T}}$ tended to increase to a greater extent in patients in whom this value at rest exceeded 30 per cent.

Effect of acetylcholine at rest. The infusion of acetylcholine resulted in no significant alteration in the mean values of alveolar or arterial oxygen tension, and the mean $\mathrm{A}$-a difference was not appreciably different. Arterial carbon dioxide tension was unchanged and physiological dead space and the ratio $V_{D} / V_{T}$ were not significantly altered by acetylcholine.

Effect of acetylcholine during exercise. The mean alveolar oxygen tension was similar to the mean values at rest and during exercise without acetylcholine. In contrast to the effect of exercise alone, the arterial oxygen tension rose slightly during exercise with acetylcholine, although the difference was not significant. The mean A-a difference therefore decreased slightly instead of increasing 
ACETYLCHOLINE AND RESPIRATORY GAS EXCHANGE IN MITRAL STENOSIS

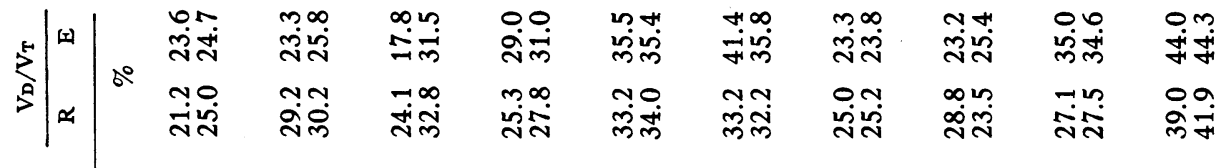

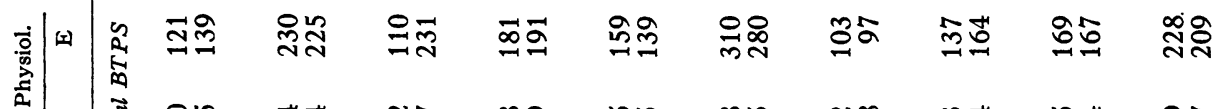

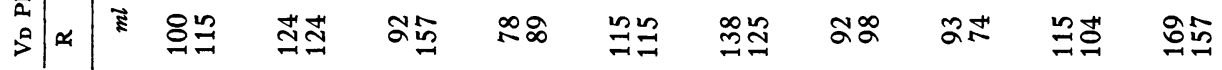

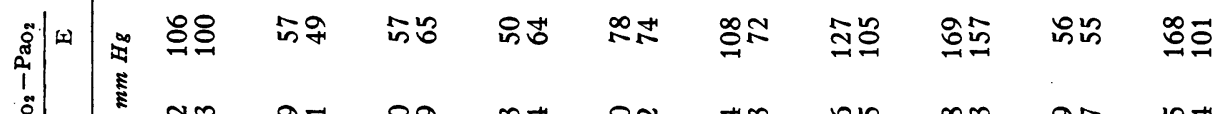
ฉँ⿱艹

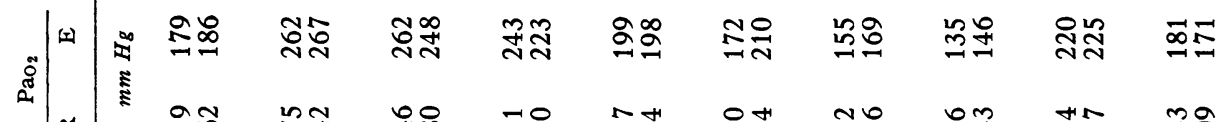
व|व

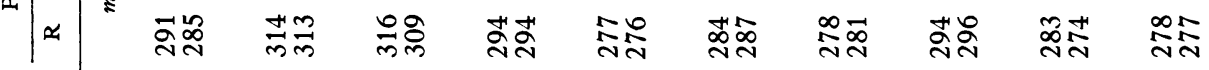
ठํ

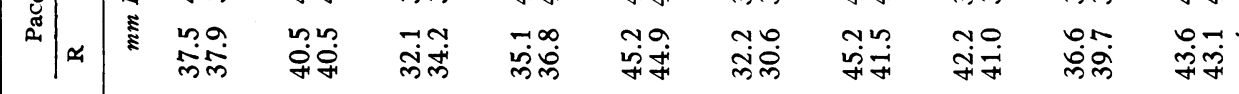

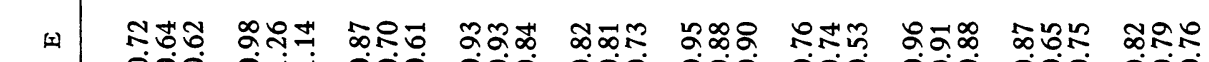
^ 000 0.7f 000 000 000 000 000 000 000 000

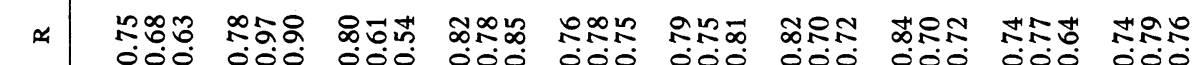
000 000 000 000 000 000 000 000 000 000

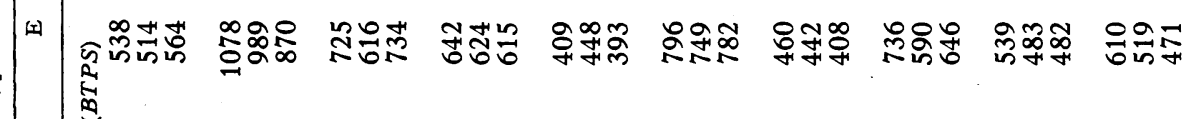

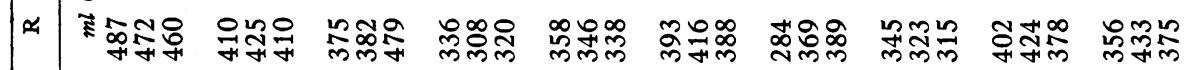

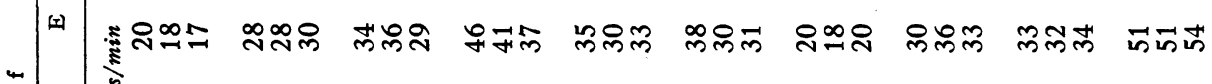
$-\infty$

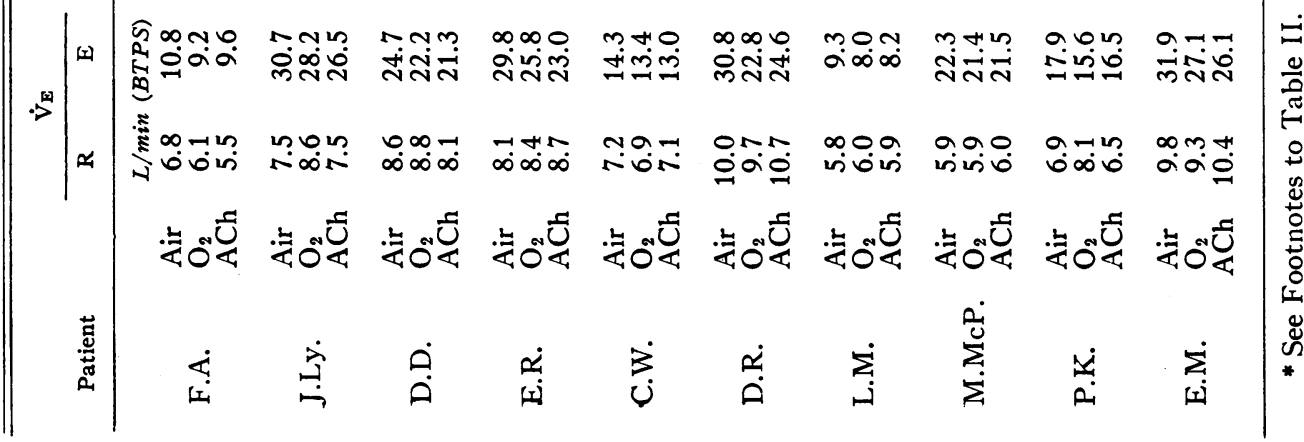




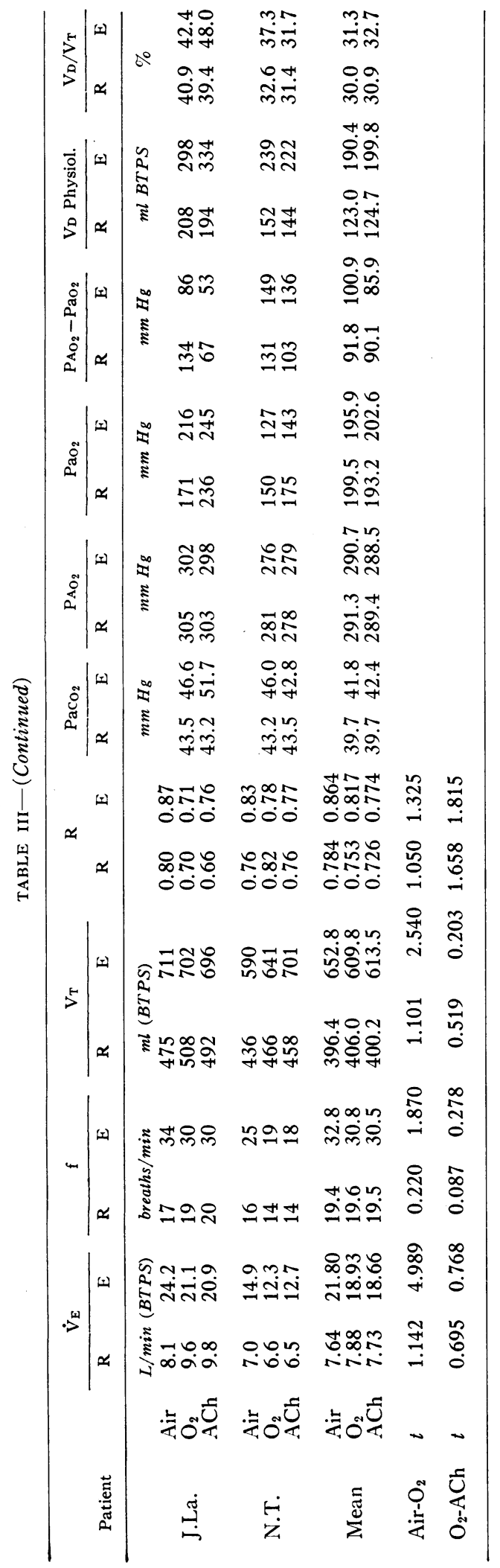

a little as occurred during exercise without acetylcholine. Arterial carbon dioxide tension increased with exercise and the increase was similar to that without acetylcholine. Physiological dead space and the $V_{D} / V_{T}$ both increased with exercise to a slightly but insignificantly greater extent than before the infusion of acetylcholine.

Correlations between derived measurements. Since there were no significant mean differences when the group of patients as a whole was considered, the data were inspected to see whether any patterns of response could be found.

A. Correlations between control values at rest. The physiological dead space increased with increasing pulmonary arterial pressure (Figure 1), but the correlation coefficient was insignificant in the present data. When data were added from an additional 12 patients, studied previously under similar conditions for another purpose, this value reached the significant value of $0.5558(\mathrm{p}<0.01)$.

There was no close correlation between the A-a difference and the physiological dead space at rest $(\mathrm{R}=0.4571)$, although larger A-a differences tended to be associated with higher values of dead space. There was no detectable correlation between A-a difference and pulmonary arterial pressure, and when data from the further 12 patients were added the correlation was only slightly improved.

$B$. Correlations between changes in A-a difference, physiological dead space and other measurements during the infusion of acetylcholine at rest. The relationship of the change in A-a difference with acetylcholine to the A-a difference at rest without acetylcholine (the latter subsequently referred to as the control value) is shown in Figure 2. Patients with a large control value tended to have a decrease with acetylcholine, while patients with a smaller control value showed an increase. Similarly, as shown in Figure 3, a large value of $\mathrm{V}_{\mathrm{D}} / \mathrm{V}_{\mathrm{T}}$ before the infusion tended to be associated with a decrease of A-a difference with acetylcholine, while the reverse occurred in patients with a lower value of $V_{D} / V_{T}(R=0.7944, p<0.01)$. There was a tendency for the A-a difference to decrease during the infusion of acetylcholine in the patients with higher pulmonary arterial pressures, while patients with lower pressures showed an increased A-a difference (Figure $4 ; \mathrm{R}=0.3232$ ). 


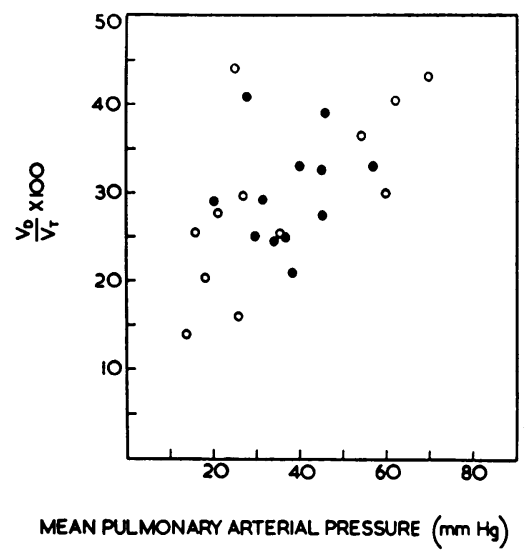

Fig. 1. The Ratio $\left(\mathrm{V}_{\mathrm{D}} / \mathrm{V}_{\mathrm{T}}\right) \times 100$ plotted against THE MEAN PULMONARY ARTERIAL PRESSURE. The measurements of $\left(\mathrm{V}_{\mathrm{n}} / \mathrm{V}_{\mathrm{T}}\right) \times 100$ were made at rest, during 47 per cent oxygen breathing. The measurements of pulmonary arterial pressure were made at rest, breathing air. The closed circles represent the patients studied in the present series. The open circles represent an additional 12 patients studied under similar conditions (see text).

The physiological dead space tended to increase during the infusion of acetylcholine when the control value for $V_{D} / V_{T}$ was small and to decrease when this value was larger, as shown in Figure 5. Physiological dead space tended to increase with acetylcholine in patients with smaller control values for A-a difference and tended to decrease in

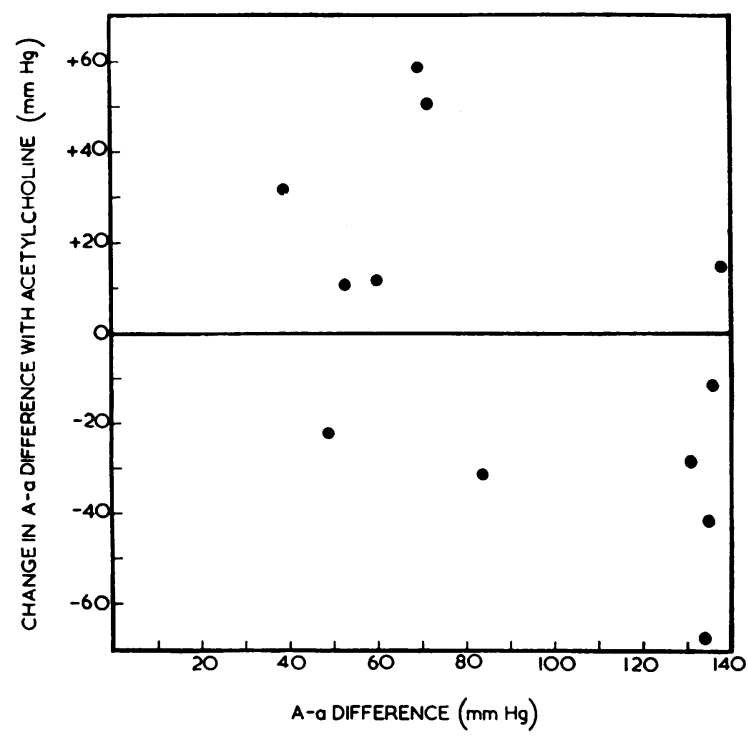

Fig. 2. Change in A-a Difference during the iNFUSION OF ACETYLCHOLINE PLOTTED AGAINST THE A-a DIFFERENCE MEASURED BEFORE THE INFUSION. All measurements taken at rest, breathing 47 per cent oxygen.

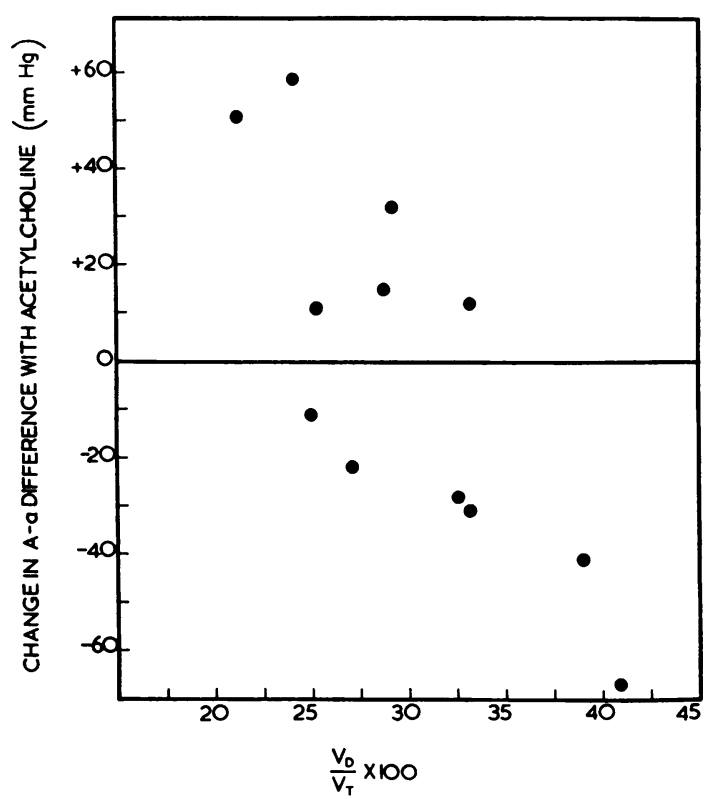

Fig. 3. Change in A-a Difference during the inFUSION OF ACETYLCHOLINE PLOTTED AGAINST THE RATIO $\left(\mathrm{V}_{\mathrm{D}} / \mathrm{V}_{\mathrm{T}}\right) \times 100$ MEASUREd BEFore THE INFUSion. All measurements taken at rest, breathing 47 per cent oxygen.

patients with larger control values for A-a difference, (Figure $6, \mathrm{R}=0.3612$ ). The correlation between pulmonary arterial pressure and the change in $V_{D}$ with acetylcholine was poor, although there was a tendency for patients with

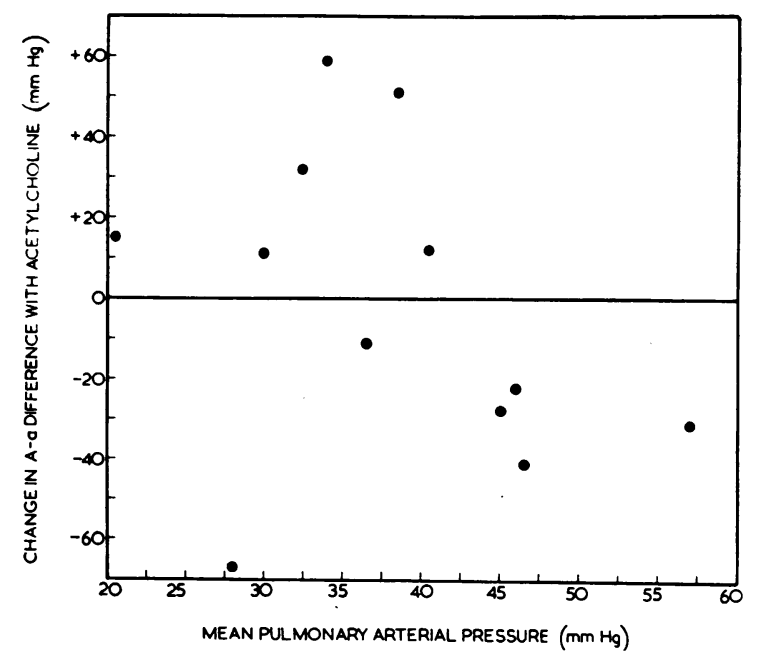

Fig. 4. Change in A-a difference during the iNFUSION OF ACETYLCHOLINE PLOTTED AGAINST THE MEAN PULMONARY ARTERIAL PRESSURE. The measurements of A-a difference were taken at rest, breathing 47 per cent oxygen. The measurements of pulmonary arterial pressure were taken at rest, breathing air. 


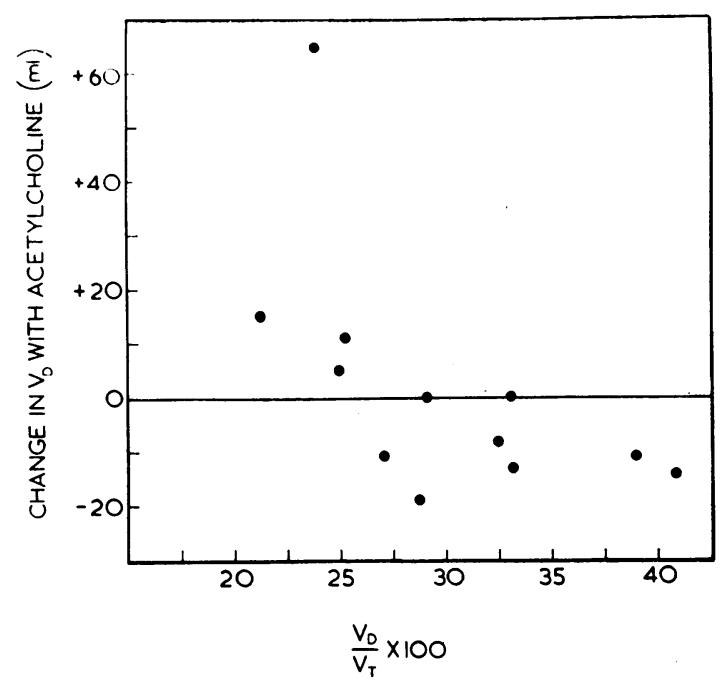

Fig. 5. Change in Physiological dead space during THE INFUSION OF ACETYLCHOLINE PLOTTED AGAINST THE RATIO $\left(V_{\mathrm{D}} / \mathrm{V}_{\mathrm{T}}\right) \times 100$ MEASURED BEFORE THE INFUSION. All measurements taken at rest, breathing 47 per cent oxygen.

higher pressures to show a decrease in dead space during infusion, while most patients with lower pressures showed an increase.

The changes in physiological dead space and A-a difference are illustrated in Figure 7, where it is clear that both tend to increase or decrease together under the influence of acetylcholine $(\mathrm{R}=$ $0.7020, \mathrm{p}<0.02$ ).

C. Correlations between changes in A-a difference, physiological dead space and other measure-

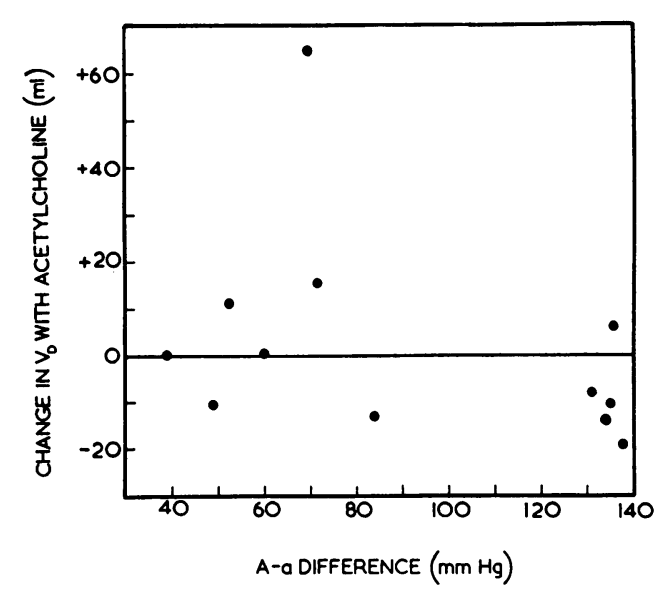

Fig. 6. Change in Physiological deAd SPACE during THE INFUSION OF ACETYLCHOLINE PLOTTED AGAINST THE A-a DIFFERENCE MEASURED BEFoRe THE INFUSION. All measurements taken at rest, breathing 47 per cent oxygen. monls during the infusion of acclylcholine during cxercise. Essentially similar correlations were found when acetylcholine was infused during exercise as were found when it was infused at rest. The A-a difference, which decreased in the group as a whole, tended to decrease to a greater extent in those patients with a high resting value for pulmonary arterial pressure, $\mathrm{V}_{\mathrm{D}} / \mathrm{V}_{\mathrm{T}}$ and A-a difference. Physiological dead space tended to decrease in patients with the higher pulmonary arterial pressures and to increase in patients in whom pressure was less elevated. Physiological dead space tended to increase in patients in whom the value at rest was normal and to decrease in pa-

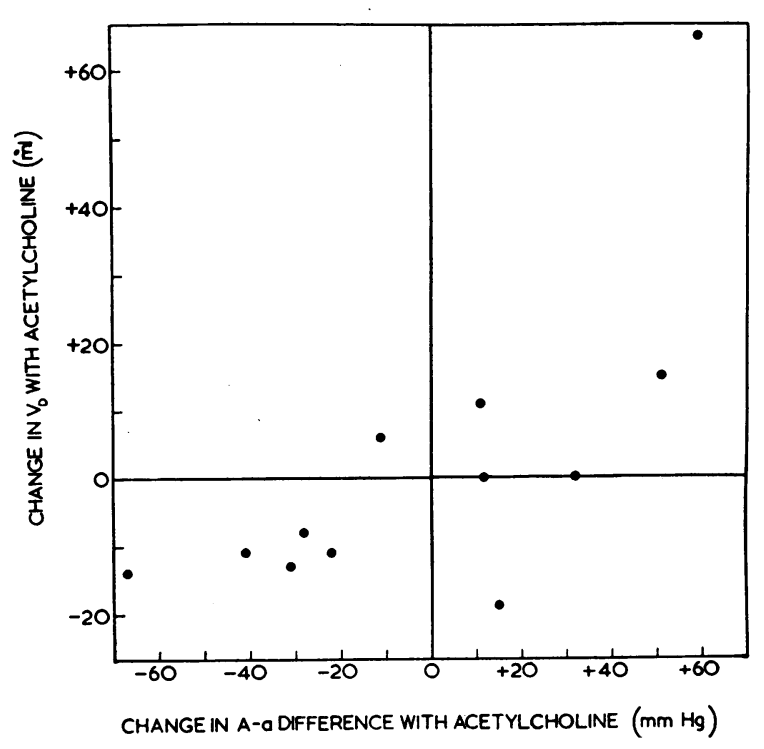

Fig. 7. Change in Physiological dead space plotted AGAINST THE CHANGE IN A-a DIFFERENCE DURING THE INFUSION OF ACETYLCHOLINE. All measurements taken at rest, breathing 47 per cent oxygen.

tients with high resting values. There was no correlation between the change in physiological dead space and the resting value for $\mathrm{A}-\mathrm{a}$ difference.

\section{Intravascular pressures, cardiac output and pulmonary ventilation}

Effects of breathing 47 per cent oxygen. The mean cardiac output, oxygen uptake and arteriovenous (A-V) oxygen difference were not significantly different when 47 per cent oxygen was breathed either at rest or during exercise. The mean pulmonary arterial pressure was similar in the two conditions at rest, but during exercise it 
was significantly lower when 47 per cent oxygen was breathed $(0.02<p<0.05)$. Pulmonary wedge pressure, obtained in seven patients only, was unaffected by breathing oxygen either at rest or on exercise, and the observed decrease in the difference between the pulmonary arterial and the pulmonary wedge pressure $(\Delta \mathrm{P})$ was not significant. The mean brachial arterial pressure was unaffected. The heart rate at rest was unchanged but during exercise it was less when breathing oxygen $(p<0.01)$. The pulmonary ventilation in the two conditions was similar at rest, but during exercise there was a significant reduction when oxygen was breathed $(p<0.01)$. This reduction resulted from a decrease in both respiratory rate and tidal volume, but only in the latter was the difference significant $(0.02<\mathrm{p}<0.05)$. The respiratory exchange ratio was insignificantly reduced during oxygen breathing.

Effects of acetylcholine, breathing 77 per cent oxygen. The cardiac output, oxygen uptake and $\mathrm{A}-\mathrm{V}$ difference were unaffected by the infusion of acetylcholine, either at rest or during exercise. The mean pulmonary arterial pressure was less during the infusion of acetylcholine both at rest and during exercise. The mean pulmonary wedge pressure in the seven patients in whom it was obtained increased slightly with acetylcholine both at rest and during exercise. In neither case was the difference significant, nor was the resultant small decrease in $\Delta \mathrm{P}$. The mean brachial arterial pressure and heart rate were not significantly altered by acetylcholine, either at rest or during exercise. Pulmonary ventilation, tidal volume and respiratory rate were unaltered, but the respiratory exchange ratio was less during the infusion of acetylcholine, although the difference was not significant.

\section{DISCUSSION}

The average value of the A-a difference in these patients at rest is more than twice the average normal value. Of the factors contributing to the total A-a difference, that due to the "membrane component" can be dismissed. Even though the patients probably had a diminished diffusing capacity for oxygen, no appreciable end-capillary gradient would exist at the alveolar oxygen tensions used in the present studies. The total A-a difference therefore consisted of a component due to anatomi- cal right to left shunting, and a component due to the inequality of ventilation-perfusion ratios in individual alveoli.

On the basis of previously published measurements in normal subjects, Farhi and Rahn (10) suggested that, at alveolar oxygen tensions such as were used in these studies, all but 1 to $2 \mathrm{~mm}$ $\mathrm{Hg}$ of the total A-a difference was due to anatomical shunting. The increased value in the patients with mitral stenosis could therefore be due to increased anatomical shunting or to the reduction in mixed venous oxygen content consequent on the reduced cardiac output. If this were the case, the anatomical shunt would represent an average of 5.1 per cent of the cardiac output, which is about twice the normal figure.

There is, however, evidence from the present studies and from other considerations that anatomical shunts do not contribute such a high proportion to the total A-a difference as appears to be the case in normal subjects. The normal anatomical venous admixture presumably consists chiefly of the drainage of bronchial venous blood into the pulmonary veins and of thebesian venous blood into the left heart. Administered into the pulmonary circulation, acetylcholine is destroyed so rapidly as not to have any effect on the systemic blood vessels, so that blood flow through bronchial and thebesian shunts would not be expected to change. There may in addition be transpulmonary shunts, but histological examination of the pulmonary vessels in patients with mitral stenosis reveals no evidence of the type of transpulmonary shunts seen in some patients with pulmonary hypertension and congenital heart disease (11). Furthermore, in order to explain the observed changes in A-a difference on the basis of altered anatomical shunting, it would be necessary to postulate a qualitatively different effect of the drug on the vessels concerned in different patients with mitral stenosis, which seems improbable.

It is hardly possible, therefore, to explain the observed changes in A-a difference solely on the basis of an alteration of the fraction of the cardiac output flowing through anatomical right to left shunts. It was observed that changes of A-a difference in either direction occurred during both exercise and the infusion of acetylcholine at rest and during exercise. Furthermore, the changes were accompanied by changes in physiological dead 
space which were significantly correlated in each situation. This suggests that an important fraction of the total A-a difference was due to unequal ratios of alveolar ventilation to pulmonary capillary blood flow $\left(\dot{\mathrm{V}}_{\mathbf{A}} / \dot{\mathrm{Q}}_{\mathrm{c}}\right)$.

The possibility that acetylcholine produced the observed effects by action on the airways with resulting changes in the distribution of ventilation must be considered. There is no published information on this point but it seems unlikely to occur in view of the destruction of the infused acetylcholine before it reaches the systemic circulation. It will also be noted that there was no observed change in total pulmonary ventilation or respiratory frequency, neither was there any alteration in the amplitude of the respiratory variation in pulmonary wedge pressure during the infusion of acetylcholine.

Söderholm and Werkö (4) suggested that acetylcholine lowered the arterial oxygen saturation by dilating preferentially those arteries which supplied underventilated areas of the lung. Even in a normal person some variation occurs in the degree of ventilation of individual alveoli. In patients with mitral stenosis this variation might conceivably be more than in normal people, although there does not appear to be any direct information on this in the literature. The effect of local underventilation of alveoli is to lower the oxygen tension in those alveoli. There is evidence that a local reduction in oxygen tension causes a local reduction in the blood flow $(12,13)$. Operating to perfection, this mechanism would result in the maintenance of equal $\dot{\mathrm{V}}_{\mathbf{A}} / \dot{\mathrm{Q}}_{\mathrm{c}}$ ratios throughout the lungs even though ventilation were unevenly distributed. The infusion of acetylcholine appears to dilate preferentially pulmonary arteries which have been constricted by hypoxia (14). Thus the effect of acetylcholine would be to increase the flow of blood through the hypoxic areas of the lung and simultaneously direct the flow of blood away from the well ventilated areas. If the patient were breathing air, this would cause a decrease in the arterial oxygen saturation, as suggested by Söderholm and Werkö. If the "distribution component" of the venous admixture contributed an important part to the A-a difference at the oxygen tensions existing in the present study, then this action of acetylcholine would be expected to increase the A-a difference and increase the physiological dead space. In those patients with a mean control pulmonary arterial pressure of less than $40 \mathrm{~mm} \mathrm{Hg}$, this is in fact what happened, and the above explanation appears plausible. It ignores the fact that it seems unlikely that any portion of the lung could be, strictly speaking, hypoxic while breathing 45 per cent oxygen. Nevertheless, the response of the pulmonary vessels to varying levels of alveolar oxygen tension may well be a graded one so that a relatively hypoxic section of the lung would still receive proportionately less blood.

The diminution in the A-a difference and in the dead space, which was caused by acetylcholine in patients with higher pulmonary arterial pressures, cannot be due to the same mechanism. It is, however, possible to explain this effect on the basis of a primary abnormality in the distribution of the flow of blood to various parts of the lung. Histologically, the smaller pulmonary arteries are not uniformly abnormal, neighboring vessels showing differing degrees of structural change. It is known that the structural abnormalities of the muscular pulmonary arteries which occur in mitral stenosis are most evident in the lower lobes (15). Angiograms also show the pulmonary arterial branches to be most affected at the lung bases (16). There is, therefore, strong evidence of a nonhomogeneity in the supply of blood to the lungs in patients with mitral stenosis and it would be reasonable to assume that this nonhomogeneity increases with increasingly severe pulmonary vascular disease. The presence of such a maldistribution of blood flow would, in the presence of a uniform or randomly uneven distribution of ventilation, give rise to an inequality of ventilation-perfusion ratios. Hence the A-a difference and the physiological dead space would be increased, and this is consistent with the observed correlation between pulmonary arterial pressure and physiological dead space. The lack of correlation between pulmonary arterial pressure and A-a difference may be the result of the variable magnitude of that portion due to anatomical shunting, which would tend to mask any relationship that might exist between pulmonary arterial pressure and the "distribution component." The structurally abnormal vessels may no longer respond to the stimulus of local variations in alveolar oxygen tensions, and this would further widen the distribution of $\dot{\mathrm{V}}_{\mathbf{A}} / \dot{Q}_{\mathbf{c}}$ 
ratios. It might be supposed that the dilating effect of acetylcholine would be strongest in those vessels in which the media had become most hypertrophied. In this case the action of acetylcholine would be to increase the flow of blood through areas with a high ventilation-perfusion ratio and to decrease the blood flow through areas with a low ventilation-perfusion ratio. Thus the effect would be to diminish the physiological dead space and to diminish the A-a difference.

The reason for the different action of acetylcholine at two different stages of the disease may therefore be seen in two overlapping processes. The first is the uneven distribution of ventilation. present to some extent at all stages. The local compensatory vasoconstriction which occurs in the underventilated areas is released by acetylcholine which therefore causes an increased A-a difference and physiological dead space. As the disease progresses, a primary maldistribution of the blood supply becomes increasingly important. Here the main action of acetylcholine is again on the more constricted vessels. In these circumstances, however, the increase in local blood supply occurs in parts of the lung irrespective of their local ventilation and alveolar oxygen tension, so that the effect is to diminish the A-a difference and dead space. With an increasing pulmonary arterial pressure the influence of a maldistribution of blood comes to predominate over the influence of a maldistribution of air, the change occurring with a mean pulmonary arterial pressure of about $40 \mathrm{~mm} \mathrm{Hg}$. Although it might appear that the present findings conflict with those of Söderholm and Werkö, it will be noted that in only one of the patients studied by these authors did the pulmonary arterial pressure exceed $45 \mathrm{~mm} \mathrm{Hg}$ at rest. Their results are not therefore inconsistent with the above hypothesis.

The hemodynamic effects of breathing 47 per cent oxygen at rest were not marked. The cardiac output was not significantly changed, and the pulmonary arterial pressure was reduced by an insignificant amount. The changes during exercise, however, were more definite and, although cardiac output was not altered, pulmonary arterial pressure and heart rate were significantly reduced. The clata are not sufficiently complete to state the mechanism of the reduced pulmonary arterial pressure with certainty, although the unchanged car- diac output with a reduction of the pressure drop across the lung suggests that oxygen acted as a vasodilator. The reduction in pulmonary ventilation during exercise is of similar degree to that observed by Cotes (17) in patients with mitral stenosis breathing 100 per cent oxygen. The infusion of acetylcholine during 47 per cent oxygen breathing did not appreciably alter cardiac output or pulmonary ventilation either at rest or during exercise. Pulmonary arterial pressure was reduced both at rest and during exercise, as were also the corresponding values for $\Delta \mathrm{P}$, although none of these changes was significant. These findings are qualitatively similar to those of Bateman, Davidson, Donald and Harris (18) for patients breathing 100 per cent oxygen.

\section{SUM MARY}

The infusion of acetylcholine in patients with mitral stenosis breathing 47 per cent oxygen produced variable changes in difference between alveolar and arterial oxygen tension (A-a difference) and physiological dead space both at rest and during exercise.

In general, A-a difference and physiological dead space either increased or decreased together. An increase was seen principally in patients with lower resting pulmonary arterial pressures, while in patients with higher pressures both quantities tended to decrease.

A hypothesis is suggested to explain these observations according to which a progressive unevenness of distribution of blood flow to the lungs develops in mitral stenosis. This finally predominates over the uneven distribution of ventilation, the adjustments to which probably account for the observed changes in the patients with less severe disease.

It was incidentally observed that breathing 47 per cent oxygen lowers the pulmonary arterial pressure, heart rate and pulmonary ventilation during exercise.

\section{ACKNOWLEDGMENTS}

Dr. J. M. Raine gave valuable assistance in a number of these studies. The work would not have been possible without the skilled technical assistance of $\mathrm{Mr}$. A. C. Pincock, Mr. R. J. Mills, Staff Nurse Margaret Booth, Miss Pauline Shaw, Miss Susan Pepper and Mrs. Anne Hollyhock. 
We gratefully acknowledge grants from the Endowment Research Fund of the United Birmingham Hospitals which assisted this work.

\section{REFERENCES}

1. Harris, P. A study of the effects of disease on the pressures in the pulmonary artery and right ventricle in man. Thesis, University of London, 1955.

2. Harris, P. Influence of acetylcholine on the pulmonary arterial pressure. Brit. Heart J. 1957, 19, 272.

3. Wood, P., Besterman, E. M., Towers, M. K., and McIlroy, M. B. The effect of acetylcholine on pulmonary vascular resistances and left atrial pressure in mitral stenosis. Brit. Heart J. 1957, 19, 279.

4. Söderholm, B., and Werkö, L. Acetylcholine and the pulmonary circulation in mitral valvular disease. Brit. Heart J. 1959, 21, 1.

5. Wade, O. L., Bishop, J. M., Cumming, G., and Donald, K. W. A method for the rapid estimation of the percentage oxygen saturation and oxygen content of blood. Brit. med. J. 1953, 2, 902.

6. Bishop, J. M. Measurement of blood oxygen tension. Proc. roy. Soc. Med. 1960, 53, 177.

7. Asstrup, P. A simple electrometric technique for the determination of carbon dioxide tension in blood and plasma, total content of carbon dioxide in plasma, and bicarbonate content in "separated" plasma at a fixed carbon dioxide tension $(40 \mathrm{~mm}$. $\mathrm{Hg}$ ). Scand. J. clin. Lab. Invest. 1956, 8, 33.

8. Sendroy, J., Jr., Dillon, R. T., and Van Slyke, D. D. Studies of gas and electrolyte equilibria in blood. XIX. The solubility and physical state of uncombined oxygen in blood. J. biol. Chem. 1934, 105, 597.
9. Standardization of definitions and symbols in respiratory physiology. Fed. Proc. 1950, 9, 602.

10. Farhi, L. E., and Rahn, H. A theoretical analysis of the alveolar-arterial $\mathrm{O}_{2}$ difference with special reference to the distribution effect. J. appl. Physiol. 1954, 7, 699.

11. Wagenvoort, C. A. The morphology of certain vascular lesions in pulmonary hypertension. J. Path. Bact. 1959, 78, 503.

12. von Euler, U. S., and Liljestrand, G. Observations on the pulmonary arterial blood pressure in the cat. Acta physiol. scand. 1946, 12, 301.

13. Dirken, M. N. J., and Heemstra, H. The adaptation of the lung circulation to the ventilation. Quart. J. exp. Physiol. 1948, 34, 213.

14. Fritts, H. W., Jr., Harris, P., Clauss, R. H., Odell, J. E., and Cournand, A. The effect of acetylcholine on the human pulmonary circulation under normal and hypoxic conditions. J. clin. Invest. 1958, 37, 99.

15. Heath, D., and Best, P. V. The tunica media of the arteries of the lung in pulmonary hypertension. J. Path. Bact. 1958, 76, 165.

16. Davies, L. G., Goodwin, J. F., Steiner, R. E., and van Leuven, B. D. The clinical and radiological assessment of the pulmonary arterial pressure in mitral stenosis. Brit. Heart J. 1953, 15, 393.

17. Cotes, J. E. The role of oxygen, carbon dioxide and lactic acid in the ventilatory response to exercise in patients with mitral stenosis. Clin. Sci. 1955, 14, 317 .

18. Bateman, M., Davidson, L. A. G., Donald, K. W., and Harris, P. A comparison of the effect of acetylcholine and $100 \%$ oxygen on the pulmonary circulation at rest in patients with mitral stenosis. In preparation. 